\title{
High Performance ALU Using Carry Look-ahead Adder
}

\author{
V.Silpa kesav ${ }^{1}$ G.Sai Srinadh ${ }^{2}$ Shaik Javeed $^{3}$ \\ Asst. Professor, CVR College of Engineering/ECE Department, Hyderabad, India \\ Email: shilpakesav@gmail.com \\ M.Tech student, CVR College of Engineering /ECE Department, Hyderabad, India \\ Email: saisrinathraju@gmail.com, \\ M.Tech student, CVR College of Engineering /ECE Department, Hyderabad, India \\ Email: javeed.shaik249@gmail.com
}

\begin{abstract}
A Low Power 8-bit Arithmetic Logic unit (ALU) using a Carry look-ahead adder (CLA) and placing Low $V_{t}$ $\left(L V_{t}\right)$ cells in Critical path is anticipated. The ALU is designed in 90nm CMOS technology. ALU is the most essential circuit in any processor. It consists of AE, LE, CLA and CE. This ALU is designed to calculate Arithmetic and Logical operations. Power and Delay values of different 8-bit adders like CLA, Sparse and Ripple Carry Adder (RCA) are designed and compared. The simulation results show that the design of ALU using CLA and incorporating High $V_{t}$ and Low $V_{t}$ cells in the CLA gives more power and delay efficient than with only Standard threshold voltage cells.
\end{abstract}

Index Terms: Arithmetic Extender, Logic Extender, Carry Extender, Carry Look-ahead Adder, ALU.

\section{INTRODUCTION}

ALU is the major power hungry block in any microprocessor and micro controller. It performs both arithmetic and logical operations. Conventional ALU consists of Arithmetic Extender, Logical Extender, Carry Extender and Ripple carry adder. An Adder is an integral part of the ALU and it is a power density block in ALU. Hence, to improve the performance of ALU in terms of Power and delay, High $V_{t}$ Cells are utilized. The three different types of transistors are

1. Low $\mathrm{V}_{\mathrm{t}}$ transistor $\left(\mathrm{LV}_{\mathrm{t}}\right)$

The low $V_{t}$ transistor type is used for applications where the speed is of primary importance. The disadvantage of this type of transistors is that, due to low threshold Voltage $\left(\mathrm{V}_{\mathrm{t}}\right)$, the static power is very high.

2. Standard $\mathrm{V}_{\mathrm{t}}$ Transistors $\left(\mathrm{SV}_{\mathrm{t}}\right)$

The standard $\mathrm{V}_{\mathrm{t}}$ transistor type is used when delay and static power has been traded off.

3. High Vth Transistor $\left(\mathrm{HV}_{\mathrm{t}}\right)$

The High Vth transistor is a favor for extremely low static power consumption.

For reducing power and delay High $\mathrm{V}_{\mathrm{t}}$ cells are used in entire ALU and in critical path of CLA, Low $V_{t}$ transistors are used. The reference [5] and [6] explains about the power reduction of the design using PTL and Gate diffusion technique and [7]-[10] shows the concept of low power techniques in various Arithmetic circuits.

Here the design of an eight bit ALU with three select lines for performing eight operations are shown in Figure-1. Out of these eight operations, four operations are logical and four operations are arithmetic operations. The design includes four basic blocks: They are CLA, Logic Extender (LE), Arithmetic Extender (AE), and Carry Extender (CE). The function of $\mathrm{LE}$ is to operate logic operations, $\mathrm{AE}$ is to operate arithmetic operations, $\mathrm{CE}$ is for carry operations and CLA is for actual arithmetic operations.

\section{CONVEnTIONAL ALU}

The Arithmetic Logic Unit (ALU) is the further most significant block in microprocessor [1]. This one is used on behalf of executing arithmetic and logic operations alike addition, Subtraction, Logical OR and Logical AND. In the Conventional ALU, Ripple carry adder (RCA) is used, and the delay and power values are more. So, instead of the RCA, Carry look-ahead adder has been selected. Since, ALU requires high speed and Low power. The overall circuit for 4-bit ALU is shown in figure1 [2]. The two different Combinational circuits in front of CLA are LE and AE.

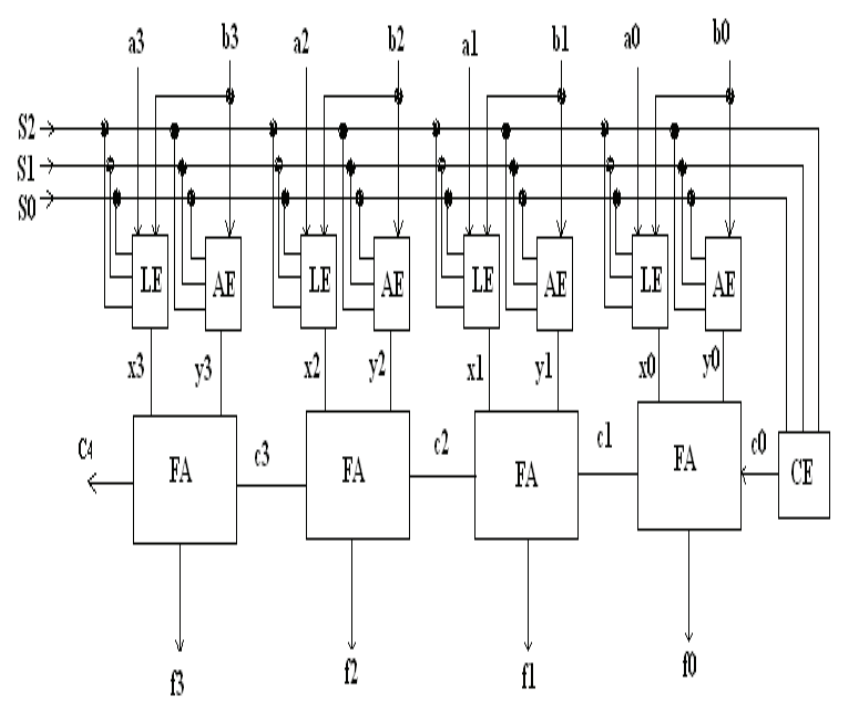

Figure 1. Existing ALU System. 
TABLE I.

Alu FunCtion TABle

\begin{tabular}{|l|l|l|l|l|l|l|l|}
\hline S2 & Sl & S0 & OperationName & Operation & $\mathrm{X}(\mathrm{LE})$ & $\mathrm{Y}(\mathrm{AE})$ & $\mathrm{CO}(\mathrm{CE})$ \\
\hline 0 & 0 & 0 & Pass & Pass A to output & A & 0 & 0 \\
\hline 0 & 0 & 1 & AND & A AND B & AAND B & 0 & 0 \\
\hline 0 & 1 & 0 & OR & A OR B & A OR B & 0 & 0 \\
\hline 0 & 1 & 1 & NOT & A $^{\prime}$ & $A^{\prime}$ & 0 & 0 \\
\hline 1 & 0 & 0 & Addition & A + B & A & B & 0 \\
\hline 1 & 0 & 1 & Subtraction & A-B & A & B $^{\prime}$ & 1 \\
\hline 1 & 1 & 0 & Increment & A+1 & A & 0 & 1 \\
\hline 1 & 1 & 1 & Decrement & A-1 & A & 1 & 0 \\
\hline
\end{tabular}

From the functional operation of ALU shown in table-1 the selection input S2 is the main important parameter for selecting Arithmetic operations and Logical operations. When the selection line S2 is ' 0 ', then Arithmetic operations are performed and when S2 is ' 1 ' then Logical operations are performed. S0 and S1 are going to select any one of the operations.

TABLE II.

TRUTH TABLE FOR LE

\begin{tabular}{|cc|c|c|}
\hline S2 & S1 & S0 & $X_{1}$ \\
\hline 0 & 0 & 0 & $a_{1}$ \\
\hline 0 & 0 & 1 & $a_{a} \cdot b_{1}$ \\
\hline 0 & 1 & 0 & $a_{1}+b_{1}$ \\
\hline 0 & 1 & 1 & $a^{\prime}$ \\
\hline 1 & $X$ & $X$ & $a_{1}$ \\
\hline
\end{tabular}

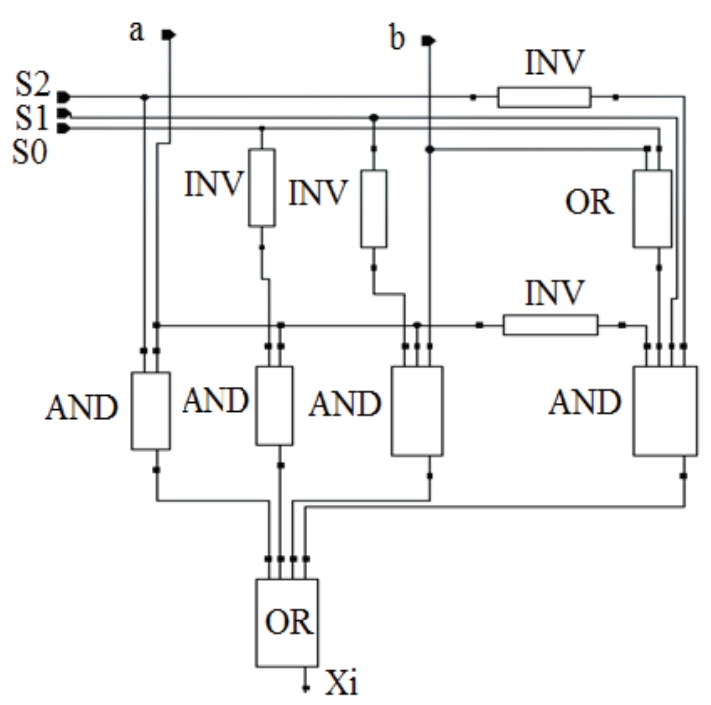

Figure 2. Schematic of LE

In LE and AE blocks all types of logical and arithmetic operations will be carried out. The operands $a_{i}$ and $b_{i}$ are inputs to LE and AE. The LE performs the operation based on selection lines (S0, S1, and S2) and inputs $a_{i}$ and $b_{i}$. The schematic diagram and truth table of LE is shown in figure2 and table- 2 respectively.
TABLE III.

TRUTH TABLE OF AE

\begin{tabular}{|l|ll|ll|}
\hline $\mathrm{S} 2$ & $\mathrm{~S} 1$ & $\mathrm{~S} 0$ & bi & $\mathrm{Yi}$ \\
\hline 0 & $\mathrm{X}$ & $\mathrm{X}$ & $\mathrm{X}$ & 0 \\
1 & 0 & 0 & 0 & 0 \\
1 & 0 & 0 & 1 & 1 \\
1 & 0 & 1 & 0 & 1 \\
1 & 0 & 1 & 1 & 0 \\
1 & 1 & 0 & 0 & 0 \\
1 & 1 & 0 & 1 & 0 \\
1 & 1 & 1 & 0 & 1 \\
1 & 1 & 1 & 1 & 1 \\
\hline
\end{tabular}

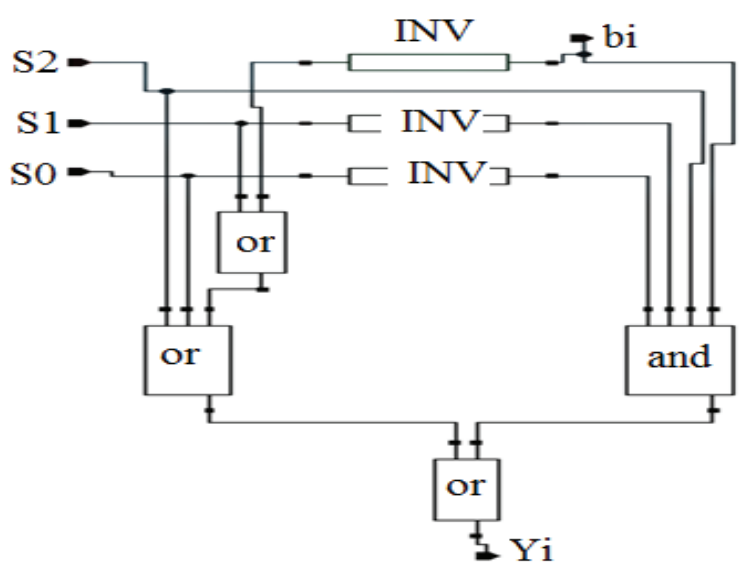

Figure 3. Schematic of AE

TABLE IV.

TRUTH TABLE FOR CE

\begin{tabular}{|cc|c|c|}
\hline S2 & S1 & S0 & C0 \\
\hline 0 & $\mathrm{X}$ & $\mathrm{X}$ & 0 \\
1 & 0 & 0 & 0 \\
1 & 0 & 1 & 1 \\
1 & 1 & 0 & 1 \\
1 & 1 & 1 & 0 \\
\hline
\end{tabular}

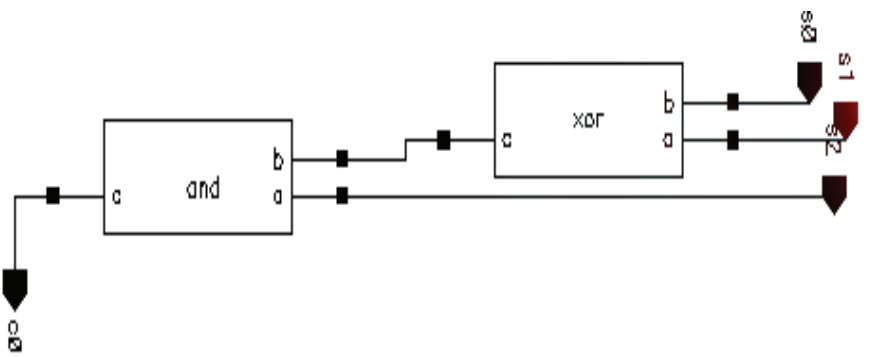

Figure 4. Schematic of CE

AE performs the operation based on selection lines and secondary input bi. It does not depend upon the primary input $\mathrm{a}_{\mathrm{i}}$. The schematic diagram and truth table of $\mathrm{AE}$ is shown in figure. 3 and table.3. The Carry Extender is another important block in ALU. It depends on selection lines and gives the output of CE to CLA. The schematic diagram and truth table of $\mathrm{CE}$ is shown in figure-4 and table-4 respectively. Now the simulation outputs of LE and AE are $\mathrm{x}_{\mathrm{i}}$ and $\mathrm{y}_{\mathrm{i}}$ respectively. These $\mathrm{x}_{\mathrm{i}}$ and $\mathrm{y}_{\mathrm{i}}$ act as inputs to the CLA and give the simulation outputs as sum and carry. The selection lines S0, S1 and S2 are three selection lines for 8bit ALU used to select outputs of LE and AE are $x_{i}$ and $y_{i}$ 
respectively. The operation of ALU through the selection lines are shown in table-I.

\section{DESIGN OF CLA}

In ripple carry adder each carry-in signal is reliant on the carry out signal from the preceding full adder. The fulladder delay is very extreme. But the carry look-ahead adder [1] doesn't depend on the previous carryout signal. The equations for Carry look-ahead adder from full adder equation [1] is

$$
C_{i+1}=x_{i} y_{i}+c_{i}\left(x_{i}+y_{i}\right)
$$

From the above equation let $g_{i}=x_{i} y_{i}$ and $p_{i}=x_{i}+y_{i}$

Then equation- (1) can be written as

$$
C_{i+1}=g_{i}+c_{i} p_{i}
$$

Using equation - (2) expand the expression for designing 4-bit Carry look ahead adder.

For getting $\mathrm{C}_{1}$ Substitute $\mathrm{i}=0$ in the equation (2),

$C_{1}=g_{0}+c_{0} p_{0}$

For $\mathrm{C}_{2}$ substitute $\mathrm{i}=1$ in the equation $-(2)$

$C_{2}=g_{i}+c_{1} p_{1}$

But $C_{1}=g_{0}+c_{0} p_{0}$, so substitute equation-(3) in the equation -(4)

Then $C_{2}=g_{1}+p_{1}\left(g_{0}+p_{0} c_{0}\right)$

$$
=g_{1}+p_{1} g_{0}+p_{1} p_{0} c_{0}
$$

For getting $\mathrm{C}_{3}$ Substitute $\mathrm{i}=2$ in the equation - (2)

$C_{3}=g_{2}+p_{2} c_{2}$

And $C_{2}=g_{i}+c_{1} p_{1}$. Hence, Substitute $C_{2}$ in the equation $-(6)$

$$
\begin{aligned}
C_{3}=g_{2}+p_{2} & \left(g_{1}+p_{1} g_{0}+p_{1} p_{0} c_{0}\right) \\
& \left.=g_{2}+p_{2} g_{1}+p_{2} p_{1} g_{0}+p_{2} p_{1} p_{0} c_{0}\right)-(7)
\end{aligned}
$$

For getting $\mathrm{C}_{4}$ Substitute $\mathrm{i}=3$ in the equation - (2)

$C_{4}=g_{3}+p_{3} c_{3}$

$$
\text { But } C_{3}=g_{2}+p_{2}\left(g_{1}+p_{1} g_{0}+p_{1} p_{0} c_{0}\right) \text {. Hence, }
$$

Substitute equation $-(7)$ in the equation $-(8)$.

$$
\text { And } C_{4}=g_{3}+p_{3}\left(g_{2}+p_{2} g_{1}+p_{2} p_{1} g_{0}+p_{2} p_{1} p_{0} c_{0}\right.
$$

$=g_{3}+p_{3} g_{2}+p_{3} p_{2} g_{1}+p_{3} p_{2} p_{1} g_{0}+p_{3} p_{2} p_{1} p_{0} c_{0}-(9)$

Using the overhead carry equations, the carry look ahead adder signals from $\mathrm{C}_{1}$ to $\mathrm{C}_{4}$ have been produced. The outputs of two input xor gates are sums of CLA. The four bit CLA is shown in Figure.5.

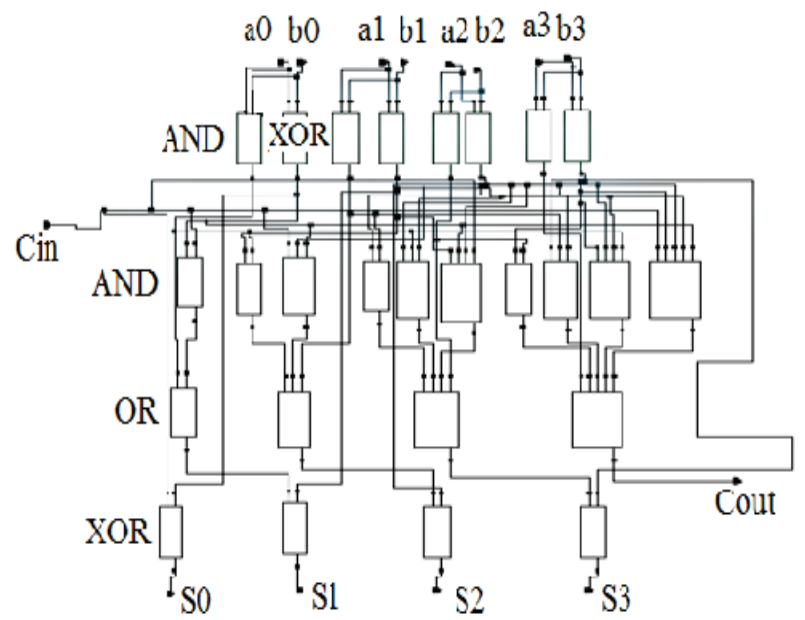

Figure 5. Schematic of 4-bit CLA

\section{PRoposed CLA}

In this paper three 8-bit Ripple carry adder (RCA), sparse adder and Carry look ahead adder are designed and the power and delay values are calculated. Table- V shows that CLA gives best power and delay values than the other two adders.

The aim of the paper is to reduce the power and delay of the ALU. So CLA has chosen instead of RCA. Since, CLA is a key block of ALU, in order to reduce power and delay, the proposed 4-bit CLA using the High $\mathrm{V}_{\mathrm{t}}$ cell concept has been shown. In the present technology, there are different types of MOS transistors. Those are Low $V_{t}$ cells, High $V_{t}$ cells and Standard $V_{t}$ cells etc. The concept of High $V_{t}$ cells is explained here.

\subsection{High $V_{t}$ Cell concept}

The region just below $V_{t}$ of a transistor is called the subthreshold region [4]. After the gate to source voltage $V_{g s}$ is less than threshold Voltage $V_{t}$, then the leakage current

Where $\mu=$ mobility

$$
\mathrm{I}_{\text {leakage }}=\mu(\mathrm{W} / \mathrm{L}) \mathrm{e}^{\left(-\mathrm{qV} \mathrm{V}_{\mathrm{t}} / \mathrm{rKT}\right)}
$$

$$
\begin{aligned}
& \mathrm{W}=\text { width of MOSFET } \\
& \mathrm{L}=\text { Length of MOSFET } \\
& \mathrm{K}=\text { Boltzmann's constant } \\
& \mathrm{T}=\text { Temperature } \\
& \mathrm{q}=\text { Charge of an electron } \\
& \mathrm{V}_{\mathrm{t}}=\text { Threshold Voltage } \\
& \eta=\text { Sub-threshold switching Coefficient }
\end{aligned}
$$

This indicates that the parameters $\mu, \mathrm{K}, \mathrm{q}$ are constants and only $\mathrm{V}_{\mathrm{t}}$ and $\mathrm{W}$ are dependent on $\mathrm{I}_{\text {leakage. As the width }}$ of MOSFET rises, leakage current also rises and as $V_{t}$ increases, the leakage current decreases exponentially. This, in turn lessens leakage power. So in this circuit all blocks of the Carry Look-ahead adder are designed and PMOS transistors are replaced with High $\mathrm{V}_{\mathrm{t}}\left(\mathrm{HV}_{\mathrm{t}}\right)$ cells. So the MOSFETs will be operated at their threshold voltage. Because of this, delay increases and power dissipation is reduced greatly.

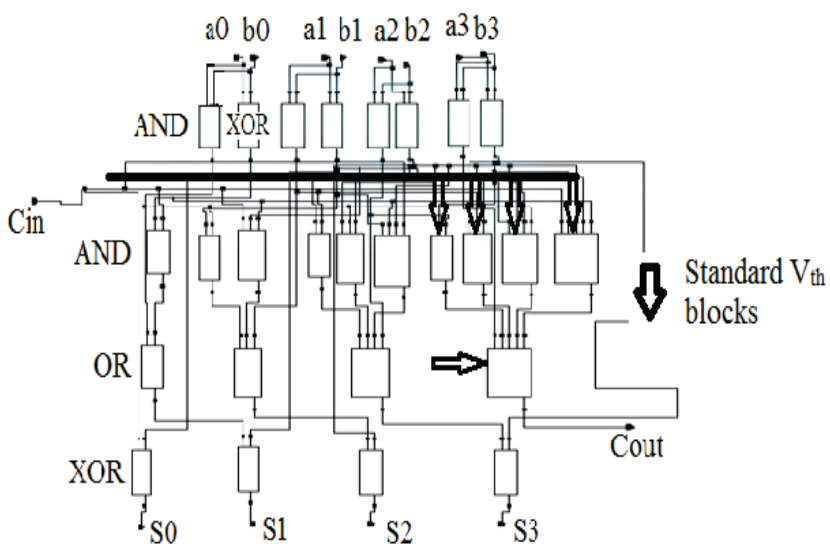

Figure 6. Proposed CLA 
This justifies the usage of high $\mathrm{V}_{\mathrm{t}}$ devices for low power applications in this design. The aim of this paper is to reduce power and delay. So the combination of these cells will give better performance than using the Standard $V_{t}$ cells. In this topic, to reduce the delay and power, using Standard $V_{t}$ cells and High $V_{t}$ cells has been explained. Hence, in this 4-bit CLA, finding the critical path is quite important. The definition of Critical path has been given below.

Critical path: The longest delay path between inputs to output.

Here, the proposed 4-bit CLA the critical path is shown in Figure.6. It is $\mathrm{C}_{\mathrm{in}} \rightarrow$ Four AND gates $\rightarrow$ OR gate. So to reduce delay, critical path blocks are designed with Low $\mathrm{V}_{t}$ cells. Next, to reduce power all remaining blocks are designed with High $\mathrm{V}_{\mathrm{t}}$ cells.

\subsection{Design of 8-bit CLA}

The proposed 8-bit CLA is designed with cascading two 4-bit CLA as shown in figure-7. The Carry out waveform of first CLA is connected to the $C_{\text {in }}$ of next CLA as shown in figure.7. The output waveform of the 8-bit CLA is shown in Figure- 8 and Figure-9

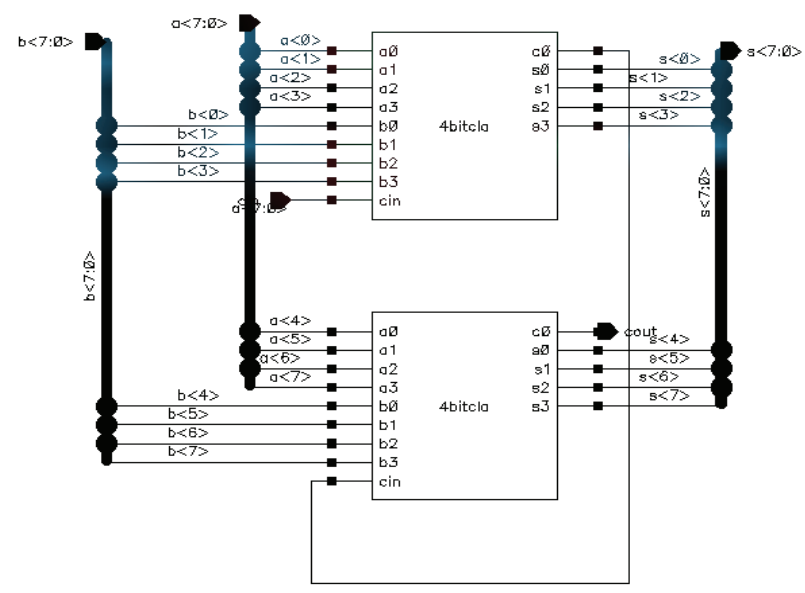

Figure 7. Schematic diagram of 8-bit CLA

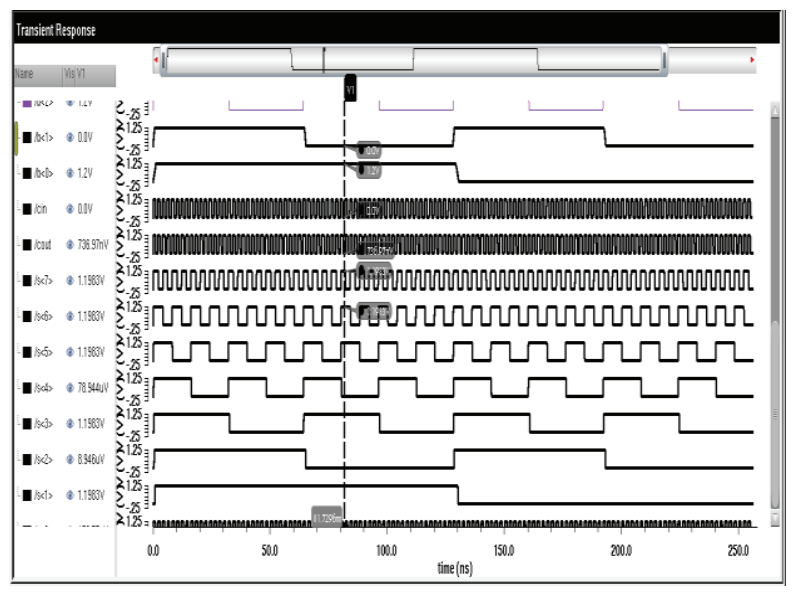

Figure 8. Simulation waveform of CLA-I

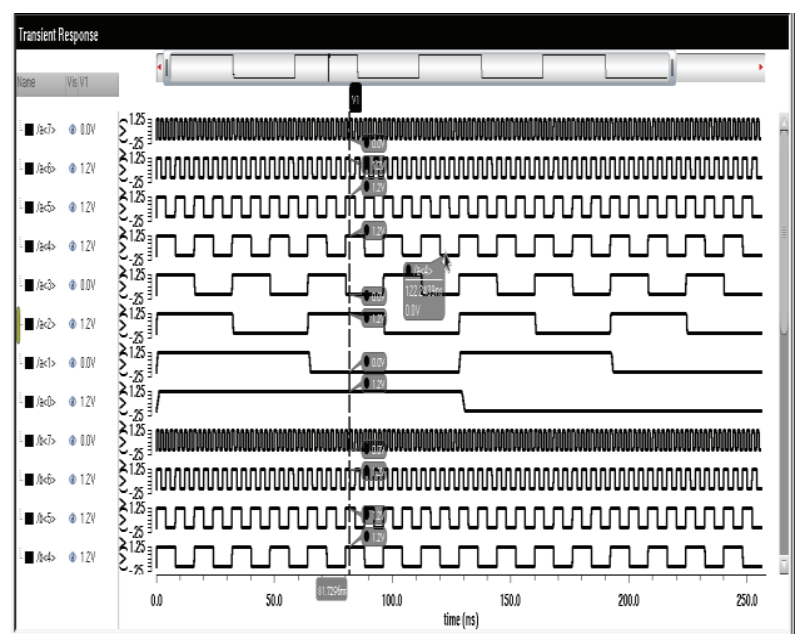

Figure 9. Simulation waveform of CLA-II

The proposed 8-bit ALU is shown in Figure.10. It has three selection lines, two 8-bit inputs $\mathrm{a}<7: 0>, \mathrm{b}<7: 0>$ and outputs are Sum $<7: 0>$ and Carry. The internal blocks are Logical Extender, Arithmetic Extender and Carry Extender respectively. The simulation waveform of 8-bit ALU is shown in figure. 11 .

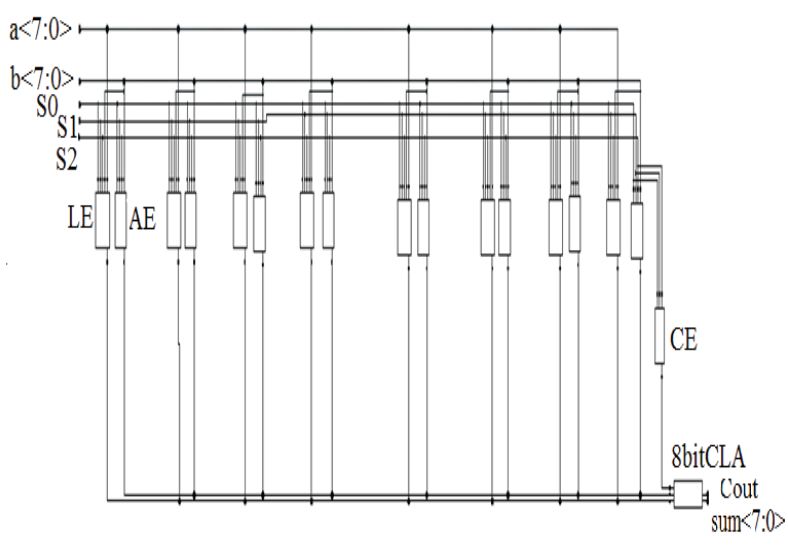

Figure 10. Proposed 8-bit ALU

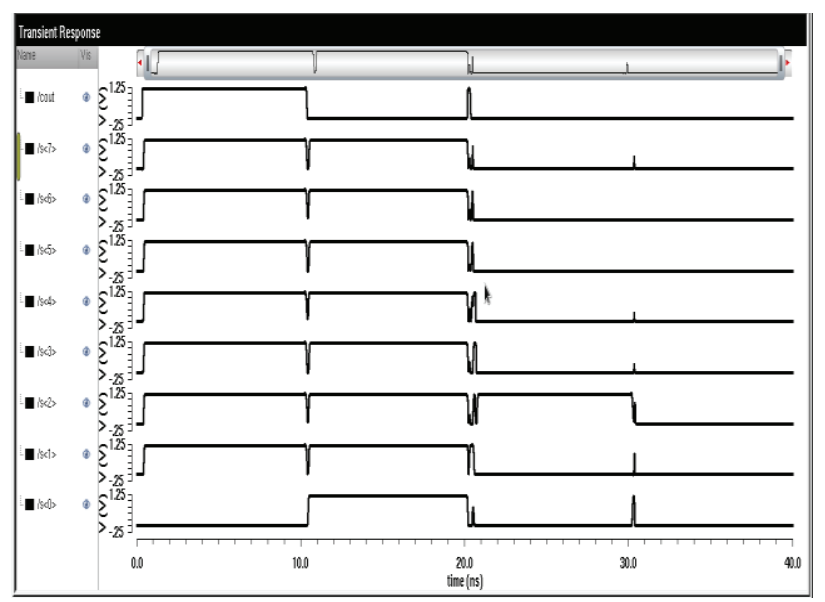

Figure 11. Simulation waveform of ALU 


\section{Simulation Results}

TABLE V.

COMPARISON TABLE OF ADDERS

\begin{tabular}{|l|l|l|}
\hline Parameter & Power $(\boldsymbol{\mu W})$ & Delay(pS) \\
\hline Ripple Carry adder & 24.22 & 64800 \\
\hline Sparse adder & 34.97 & 62400 \\
\hline Carry Look- ahead adder & 22.92 & 81.7 \\
\hline
\end{tabular}

Table $-\mathrm{V}$ shows the power and delay values of the Ripple Carry adder, sparse adder and Carry look-ahead adder. In the above mention adders Carry Look ahead adder gives Low power and delay.

TABLE VI.

PERFORMANCE PARAMETERS OF CARRY LOOK-AHEAD ADDER

\begin{tabular}{|l|l|l|}
\hline Parameter & Power $(\boldsymbol{\mu W})$ & Delay(pS) \\
\hline Using Standard Vt Cells & 5.619 & 89.13 \\
\hline Using High Vt Cells & 3.81 & 136.9 \\
\hline $\begin{array}{l}\text { Applying Low Vt cells } \\
\text { in Critical Path }\end{array}$ & 3.92 & 102.2 \\
\hline
\end{tabular}

Table.VI shows that the performance of CLA using Standard $V_{t}$ cells, using High $V_{t}$ cells and after applying Low $V_{t}$ cells in the Critical path. The average power dissipation of the Carry look-ahead adder (CLA) is $5.61 \mu \mathrm{W}$. After applying High $\mathrm{V}_{\mathrm{t}}$ cells in all P-MOSFETs, power consumption was reduced to $3.81 \mu \mathrm{W}$ and delay was increased to $136.9 \mathrm{pS}$. I.e. $47 \%$ of power consumption was reduced and $53 \%$ of delay was increased. In this ALU design, delay is also an important parameter. So, further reducing the delay, critical path was identified and placed in all the cells in the critical path to Standard $V_{t}$ cells. Then $2.8 \%$ of power consumption was increased and $33.9 \%$ of delay was reduced. Hence, this high performance of CLA is used in the ALU.

TABLE VII

PERFORMANCE OF 8 BIT ALU

\begin{tabular}{|c|c|c|}
\hline Parameter & Power $(\boldsymbol{\mu W})$ & Delay(pS) \\
\hline $\begin{array}{c}\text { 8bit ALU using } \\
\text { Standard V Cells }\end{array}$ & 50.8 & 246.3 \\
\hline $\begin{array}{c}\text { 8 bit ALU using High } \\
\mathrm{V}_{\mathrm{t}} \text { cells in CLA }\end{array}$ & 44.32 & 296.24 \\
\hline $\begin{array}{c}\text { 8bit ALU Applying } \\
\text { Low } \mathrm{V}_{\mathrm{t}} \text { in Critical Path of } \\
\text { CLA }\end{array}$ & 43.58 & 258.5 \\
\hline
\end{tabular}

Table-VII shows the performance of 8 bit ALU when it is designed with Standard $V_{t}$ cells, High $V_{t}$ cells and after applying Low $\mathrm{V}_{\mathrm{t}}$ cells in the Critical path of CLA. The average power consumption and delay of ALU is $50.8 \mu \mathrm{W}$ and 246.3pS respectively. After placing $\mathrm{HV}_{\mathrm{t}}$ cells in CLA $14.6 \%$ of average power consumption was reduced and $20 \%$ of delay was increased. So, to further reduce the power and delay Low $\mathrm{V}_{\mathrm{t}}$ cells are placed in critical path of CLA. Then $1.69 \%$ of power consumption was reduced and $14.5 \%$ of delay was reduced. This shows the best performance of power and delay of 8-bit ALU.

\section{Conclusions}

The main goal of this paper is to reduce power and delay. The key element in the 8-bit ALU is the 8-bit Carry lookahead adder. So, to reduce power High $\mathrm{V}_{\mathrm{t}}$ cells are used in the P-MOSFET's of Logic gates, and to reduce delay standard $V_{t}$ Cells are used in logic gates of critical path. After applying the High $\mathrm{V}_{\mathrm{t}}$ Cell concept in CLA, power reduction in ALU was $14.6 \%$, and the delay reduction was $14.5 \%$ after placing Low $\mathrm{V}_{\mathrm{t}}$ cells in the Critical path of CLA. This 8-bit ALU can be designed for other than these eight operations. This ALU can be extended to 16-bit also. This 8-bit ALU is can operate all Arithmetic and Logical operations. The total 8-bit ALU is designed in 90nm CMOS technology using Cadence tools.

\section{REFERENCES}

[1] Microprocessors Design Principles and Practices with VHDL by Enoch .O.Hwang.

[2] R.Durga Bhavani, V. Silpakesav "Efficient design of Low power 4 bit ALU using HVT Cell Concept" in CVR Journal of Science and Technology, Volume 9, December 2015, ISSN 2277-3916, pp-61-65., India.

[3] T. Ester Rani, Dr. M. Asha Rani, Dr. Rameshwarrao, "Area Optimized Low Power Arithmetic and Logic Unit", $3^{\text {rd }}$ International Conference on Electronics Computer Technology (ICECT 2011), V3-224-228.

[4] Silpakesav, K.S. Nayanathara, B.K.Madhavi.: 'Design of Low Power SAR ADC for ECG Using 45nm CMOS', International Journal of VLSI design \& Communication Systems (VLSICS) Vol.8, No.1, pp. 19-29, February 2017.

[5] P.Satyamoorthy, S.Vijya Lakshmi and A.Daneil Raj "Efficient Design of Low power ALU using PTL-GDI Logic Full adder", in IJCA Dec 2013.

[6] Lakshmi Swetha and K.Kalpana "An Efficient Design and Implementation of ALU using Gated Diffusion Index", in IJECS Volume 4 Issue 5 May 2015.

[7] Amit Singh Gaur, Jyoti Budakoti, "Energy Efficient Advanced Low Power CMOS Design to reduce power consumption in Deep submicron Technologies in CMOS circuit for VLSI Design", IJARCCE, Vol.3, Issue 6, June 2014.

[8] N.Weste and K.Eshragian, principles of CMOS VLSI Design: A Systems Perspective, Pearson/Addison Wesley Publishers, 2005.

[9] P.Chandrakasan, S.Sheng, and R.W.Broderson, "Low-Power CMOS digital design", IEEE Solid- state circuits, Vol.27, pp.473-483; April.1992.

[10] Bellaouar.A and Elmasry.M,'Low-Power Digital VLSI Design: Circuits and Systems Boston, Massachusetts: Kluwer Academic Publishers, 1995. 